\title{
A photonic crystal fiber splice with a standard single mode fiber
}

\author{
Michał Murawski, ${ }^{*}$ Leszek R. Jaroszewicz, and Karol Stasiewicz \\ Faculty of New Technologies and Chemistry, Military University of Technology, \\ Gen. Sylwestra Kaliskiego 2, 00-908 Warszawa
}

Received September 28, 2009; accepted September 29, 2009; published September 30, 2009

\begin{abstract}
We report on the optimization of a fused splice between a photonic crystal fiber (PCF) with a standard single mode fiber (SMF) made by the filament fusion with continuous laser illumination. For the SMF•28 connection with a PCF having a similar mode field diameter splice losses are lower than $0.40 \mathrm{~dB}$ in comparison with the reported dependences in a spectral range of $1.51-1.63 \mu \mathrm{m}$. Based on the results obtained two aspects of splice are discussed: the mentioned above wavelength dependences as well as an observed nonreciprocal phenomenon according to light propagation through the splice. The discussed data have shown that such a SMF-PCF splice is suitable for the construction of a patch cord for measurement devices.
\end{abstract}

Generally, conventional fusion splice technologies are useless for a photonic crystal fiber (PCF). This is primarily because PCFs derive their waveguiding characteristics from fine air-holes that are inevitably altered, oven collapsed, by high temperatures of conventional fusion splicing [1]. Moreover, the application of a PCF with commercial available optical sources requires a low-loss fusion splice with a conventional single mode fiber (SMF) in which a mode field diameter (MFD) is generally different from the MFD of a PCF according to their sizes and the fact that the fibers have a different geometrical shape of guiding beams.

Numerical calculations based on early experiments [2] suggested that SMF-PCF splice losses as low as $0.20 \mathrm{~dB}$ could be achieved [3], where losses are estimated as an area cover integral [4]:

$$
\alpha_{T}=-10 \log \left(\left|\int_{-\infty}^{\infty} \int_{-\infty}^{\infty} \Psi_{1}(x, y) \Psi_{2}(x, y) d x d y\right|^{2}\right)
$$

where $\Psi_{1}$ and $\Psi_{2}$ are the modal fields of the two splicing fibers. It is easy to see that this fundamental estimation for losses in a splice fulfils the reciprocal condition according to the direction of light propagation through a splice.

However, practical results are still far from theoretical consideration and different techniques are proposed. The applied GRIN fiber lenses give losses up to $0.40 \mathrm{~dB}$

\footnotetext{
*E-mail: jarosz@wat.edu.pl
}

between the same PCFs and $0.44 \mathrm{~dB}$ for the PCF-SMF connection having a similar MFD [5]. For splicing SMFs with small-core PCFs the losses equal to $0.90 \mathrm{~dB}$ (PCF with $4.1 \mu \mathrm{m}$ MFD) by repeated arc discharges were reported [6]. Finally, we have reported on the splicing polarization maintaining an SMF with a hollow-core PCF with losses equal to $0.62 \pm 0.24 \mathrm{~dB}$ applying the continuous laser illumination of the splice prepared on arc fusion machine [7]. It should be noticed that such a splice characterizes a nonreciprocal phenomenon which gives losses equal to $2.19 \pm 0.33 \mathrm{~dB}$ for the return propagation which is caused by the mode evolution in a hollow-core PCF.

In this letter a further improvement in the splice technology designed for the connection of a PCF with an SMF has been presented. For the splice preparation a Vytran FFS-2000 splicer (see Fig. 1) has been used employing the filament fusion instead of the arc fusion. Because a standard fusion procedure involving a splicer is not suitable for specific parameters of different photonic and standard fibers, a technique based on the application of continuous laser illumination while preparing a splice has been applied according to the procedure described below.

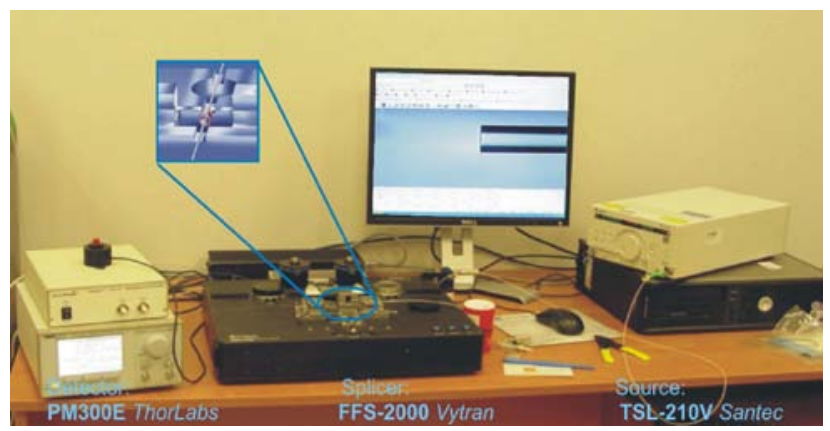

Fig. 1. General view of a set-up for fibers splicing in continuous laser illumination

A classical patch cord of an SMF• 28 fiber about $2 \mathrm{~m}$ long was connected through an FC/APC to the output of a tunable laser (TSL-210V). The laser was run continuously during the splice procedure with an output power of $10 \mathrm{~mW}$. The normal-cleaved SMF and PCF 
were placed into the FFS-2000 splicer, and manual-mode translational and rotational alignment was performed in order to maximize laser transmission through the fiber joint which was detected by a PM300E detector. PCFs of lengths of $1.5-2 \mathrm{~m}$ were used in the whole experiment to fuse with the above SMF patch cord. Splice loss was calculated from laser transmission through the spliced patch cord SMF-PCF assembly, taking into account the attenuation in the given length of a PCF. Such an approach gives splice losses estimation as power decreasing on a fiber output [8]:

$$
\alpha_{E}=-10 \log \left(\frac{P_{\text {out }}}{P_{\text {in }}}\right)
$$

where $\mathrm{P}_{\text {out }}, \mathrm{P}_{\text {in }}-$ output and input power in the fiber, respectively.

The SMF and PCF with a similar numerical aperture (NA) and MFD for $1.55 \mu \mathrm{m}$ have been used for spice optimization. From this reason the index-guiding pure silica PCF (LMA•8, Crystal Fibre) with a core diameter of $8.5 \pm 0.3 \mu \mathrm{m}$, MFD equal to $6.0 \pm 1.0 \mu \mathrm{m}$ and NA equal to 0.19 at $1.55 \mu \mathrm{m}$, has been used for join with SMF.28. The MFD and NA of the SMF.28 are $10.4 \mu \mathrm{m}$ and 0.14 at $1.55 \mu \mathrm{m}$, respectively.

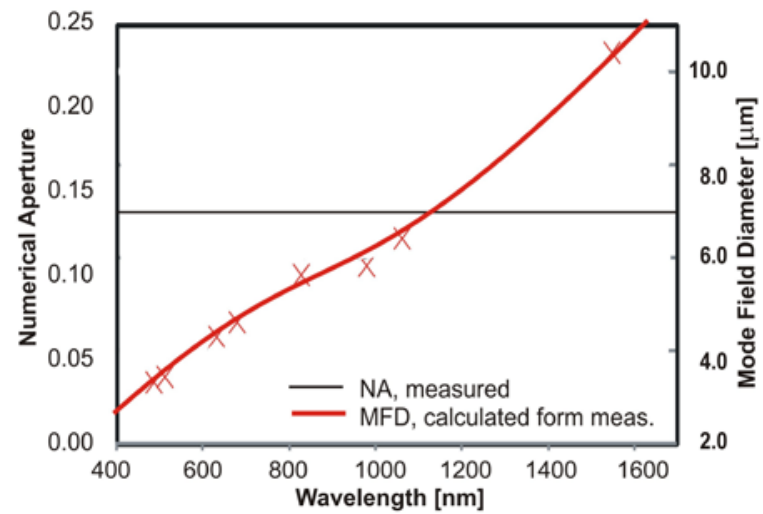

a)

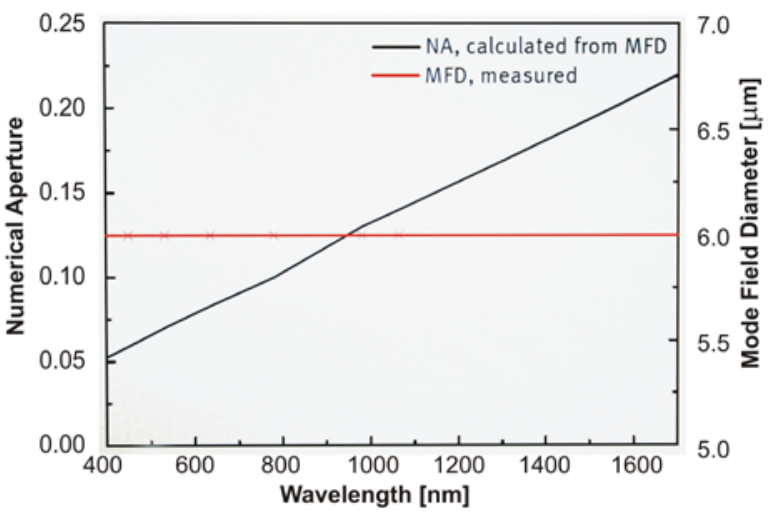

Fig. 2. Spectral dependences of MFD and NA for two classes of fibers: a) SMF•28 [9], and b) LMA•8 [10]
However, it should be noticed that a photonic crystal fiber has generally different spectral dependences of these parameters from a standard single mode fiber, as shown in Fig. 2.

The application optimized parameter for splicing [11] yields a coupling loss equal to $0.30 \pm 0.05 \mathrm{~dB}$ at $1.55 \mu \mathrm{m}$ for SMF and PCF connection, as shown in Fig. 3.

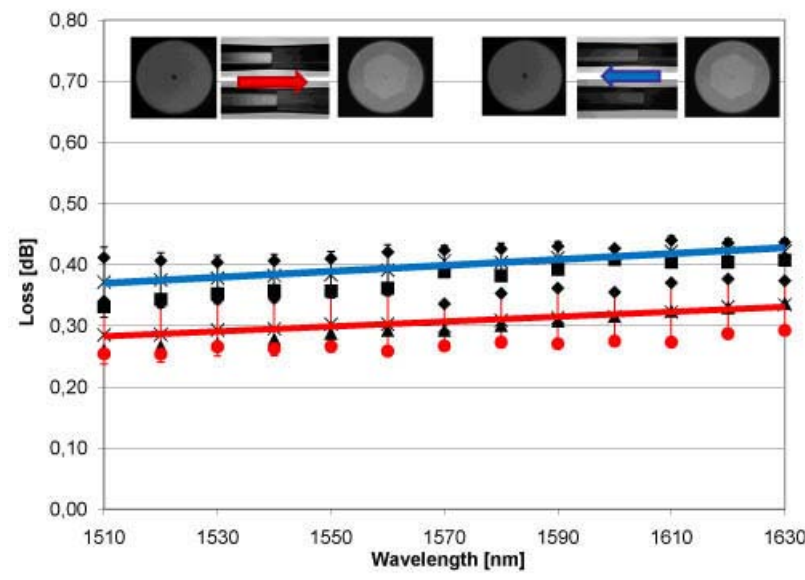

Fig. 3. Spectral characteristics of splicing losses between SMF•28LMA $\bullet 8$ for the light passes in direction SMF-PCF (red colour curve) and PCF-SMF (blue colour curve)

Optimized splices according to low losses need the taper technology for the adjustment of the MFDs of two joined fibers. The application of a pre-fusion process performed with the FFS-2000 provided an appropriate taper with a $10 \%$ waist in the splice region, as shown in the top of Fig. 3. It should be noticed that such a splice has a spectral losses dependence, and changes from $0.29 \pm 0.05 \mathrm{~dB}$ to $0.33 \pm 0.04 \mathrm{~dB}$ for wavelength changes in a range of 1.51-1.63 $\mu \mathrm{m}$ (red colour curve in Fig.3). The fusion process generates the same small separation between two fiber faces. Even if fiber parameters are optimized for a given wavelength (Fig. 4a), different behaviour of splicing fibers shown in Fig. 2 has influence on generation spectral dependence of losses according to the scheme shown in Fig. $4 \mathrm{~b}$.

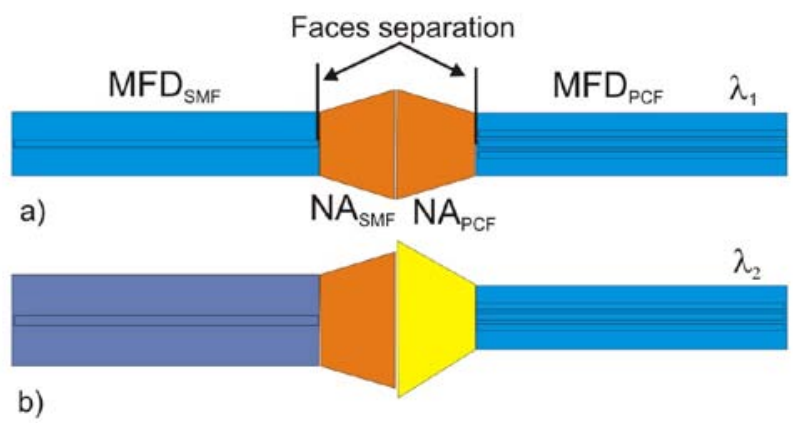

Fig. 4. Scheme of a generation spectral dependence of splicing losses: a) optimized connection for a given wavelength $\lambda_{1}$, b) structures change observed for another wavelength $\lambda_{2}\left(\lambda_{1}<\lambda_{2}\right)$ 
Moreover, coupling losses higher than $0.09 \mathrm{~dB}$ for the opposed light propagation (blue colour curve in Fig. 3) has been found. This phenomenon is connected with a method of splice manufacturing in continuous laser illumination, where splice optimization is achieved for the maximum output power and splice losses estimation is made according to formula (2). For fibers with different MFDs and NAs such an approach cannot fulfil the reciprocal condition for losses estimation according to the direction of light propagation through a splice. As one can see from the scheme presented in Fig. 4b the power coupling is different for two directions. Hence, the reciprocal PCF-to-SMF splice loss was found to be $0.39 \pm 0.04 \mathrm{~dB}$ at $1.55 \mu \mathrm{m}$, which is caused by mode evolution in a PCF.

The final characteristic of the patch cord made up by a $1.5 \mathrm{~m}$ long LMA•8 photonic crystal fiber placed between two pieces of $\mathrm{SMF} \cdot 28$ with FC-APC connectors on two patch cord ends is shown in Fig. 5. As one can see, the total coupling loss is about $0.60-0.70 \mathrm{~dB}$ for the considered spectral range.

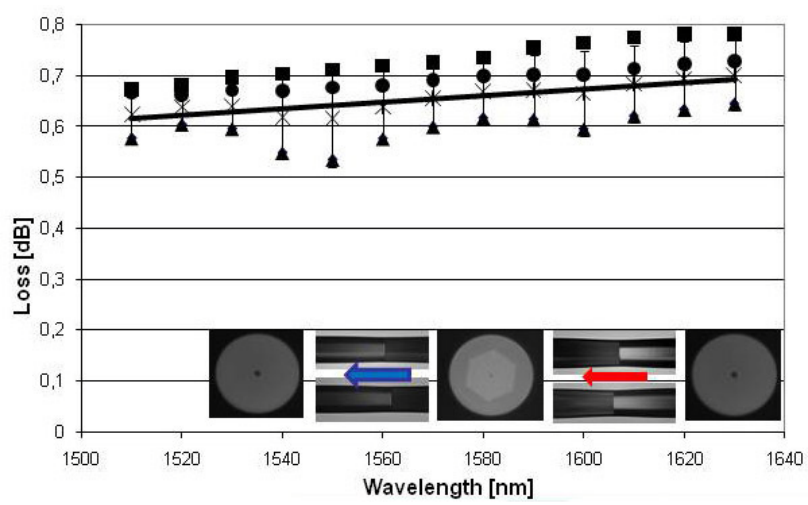

Fig. 5. Spectral characteristics of the splicing losses in the SMF•28-toLMA $\bullet$-to-SMF•28 patch cord

Reported results show the possibility of making SMFPCF patch cords for practical application in measurement devices. In our opinion such elements are fundamental for practical application any photonic crystal fiber. The presented technology based on a filament fusion splicer provides a low-losses joint especially for joint the fibers with similar MFDs (less than $0.50 \mathrm{~dB}$ ). It has also been shown that connection of SMF with different PCFs shows wavelength dependence of the losses as well as directional properties. The nonreciprocal phenomena are important for sensors application of the PCF, where short or very short length of PCF is used as sensors' transducer. In such approach low-loss splice can be connected with existence cladding modes and their elimination by conventional method has not been used because needs longer distance of fiber. Hence this phenomenon should be taken under consideration in sensor construction.
This work has been financially supported by the Polish Ministry of Sciences and Higher Education, Key Project POIG.01.03.01-14-016/08 "New Photonic Materials and Their Advanced Applications" and within the framework of the EU Action COST-299 "FIDES”.

\section{References}

[1] B. Bourliaguet et al. Opt. Express 11, 3412 (2003).

[2] P.J. Bennet et al. Opt. Lett. 24, 1203 (1999).

[3] J.T. Lizier, G.E. Town, IEEE Photon. Techn. Lett.. 13, 794 (2001).

[4] R.K Sinha, K.V. Sailendra, SPIE 4655, 296 (2002).

[5] A.D. Yablon, R.T. Bise, IEEE Photon. Techn. Lett.. 17, 180120 (2005).

[6] L. Jin W. Xiao, M.S. Demokan, Opt. Lett. 32, 115 (2007).

[7] J.T. Kristensen et al. Opt. Express 16, 9986 (2008).

[8] L. Dong et al. Opt. Express 16, 16423 (2008).

[9] Corning ${ }^{\circledR}$ SMF-28 ${ }^{\mathrm{TM}}$ CPC6 Single-Mode Optical Fiber, Product Information, http://www.corningfiber.com

[10] Large Mode Area Photonic Crystal Fiber LMA-8, Product Information, http://www.crystal-fibre.com

[11] L.R. Jaroszewicz, M. Murawski, K. Stasiewicz, and P. Marć, Proc. SPIE 7503, 214 (2009) in press 